\title{
Particle Swarm Optimization Algorithm Establish the Model of Tobacco Ingredients in Near Infrared Spectroscopy Quantitative Analysis
}

\author{
Bibo $\mathrm{Ma}^{1,2, *}$ and Haiyan $\mathrm{Ji}^{1,2, * *}$ \\ ${ }^{1}$ College of Information and Electrical Engineering, China Agricultural University, \\ Beijing 100083, China \\ ${ }^{2}$ Key Laboratory of Modern Precision Agriculture System Integration Research, \\ Ministry of Education, China Agricultural University, Beijing, China 100083 \\ Mabibo1986@163.com, instru@cau.edu.cn
}

\begin{abstract}
. 57 tobacco samples were chosen, the near-infrared diffuse reflectance spectra at five different parts of tobacco leafs were averaged as the original spectra, the range of 4000-9000 wavenumber of spectral information was selected after wavelength selection, first-order differential was used to eliminate the impact of offset, PCA was used to reduce the dimensions and select the most representative of the principal components of the sample information. After these, use PSO and the data of chemical composition of nicotine and total nitrogen, to establish the models of near-infrared spectra of the main ingredient in tobacco quantitative analysis. In which, nicotine and total nitrogen quantitative analysis of modeling set correlation coefficients were $0.8886,0.9290$; the prediction set correlation coefficients were $0.8786,0.8487$. RMSEC values were $0.4737,0.2215$; RMSEP values were $0.6417,0.2677$. The results show that: PSO can be used to establish the model of nicotine and total nitrogen by near infrared spectroscopy quantitative analysis in tobacco.
\end{abstract}

Keywords: Tobacco, Particle Swarm Optimization (PSO), Principal Component Analysis (PCA), Near infrared spectroscopy.

\section{Introduction}

Near infrared reflectance spectroscopy does not require any chemical pretreatment for the sample. Compared with traditional chemical analysis and other spectroscopy methods, it has many advantages, such as cheap, convenient, fast and non-destructive characteristics[1].

Currently, there are some methods in the near-infrared spectroscopy quantitative analysis, such as partial least squares (PLS), support vector machines (SVM) and artificial neural network (ANN). In 2007, Yan Qing Duan, Jia Jun Wang, and others use PLS method for quantitative analysis for the chemical composition of five kinds of

\footnotetext{
${ }^{*}$ Research field: Spectral analysis.

${ }^{* *}$ Research field: Spectral analysis and sensing technique.

D. Li and Y. Chen (Eds.): CCTA 2012, Part II, IFIP AICT 393, pp. 92-98, 2013.

(C) IFIP International Federation for Information Processing 2013
} 
tobacco sheet .The results showed that: near-infrared predictive value and the measured values have a good correlation, the model has better prediction accuracy[2] ; In 2007, $\mathrm{Xu}$ Liu, Hua Chen use the PCA-SVR and the ANN for a quantitative analysis of total sugar, reducing sugar, total nitrogen and nicotine content in tobacco. The results show that: the SVR model has a better prediction[3] ; In 2009, Yong Zhang, Qian Cong use the SVM for a quantitative analysis of total sugar, reducing sugar, nicotine and total nitrogen in tobacco. The results show that: the SVM model's classification accuracy rate can up to $100 \%$, the quantitative analysis of four components of the prediction coefficient of determination $\left(\mathrm{R}^{2}\right)$, the prediction mean square error (RMSEP) and the average relative error (RME) show that: the model has a better prediction [4].

In this paper, PSO method is used in near-infrared spectroscopic quantitative analysis of total nitrogen and nicotine content in tobacco, in order to prove that the PSO method can be used in the near-infrared quantitative analysis in tobacco.

\section{The Basic Principles}

\subsection{The Basic Principle of PSO}

PSO, the English name is Particle Swarm Optimization, is proposed by Kennedy and Eberhart in the study of birds and fish group's behavior in 1995. It's a swarm intelligence algorithm [5]. In PSO, each optimization problem can be a bird in the search space (we can call it a particle). All the particles have an optimized to determine the fitness value by a function and have a speed to determine their direction and speed for flying.

Firstly, the algorithm initializes a group of random particles, and then finds the optimal solution by iteration. In each iteration, the particles update their speed and position by individual extreme and global extreme. The algorithm assumes that all particles are moving in the personal best position and global best position, and mark $x_{i}=\left(x_{i 1}, x_{i 2} \ldots x_{i n}\right)$ to initialize the position of the particle, mark $v_{i}=\left(v_{i 1}, v_{i 2} \ldots v_{i n}\right)$ to initialize the velocity, mark $p_{i}=\left(p_{i 1}, p_{i 2} \ldots p_{i n}\right)$ as the best result for searching so far for the particle of $\mathrm{i}$, mark $p_{g}=\left(p_{g 1}, p_{g 2} \ldots p_{g n}\right)$ as the best result for searching so far for the all particles. In each iteration, the particles update themselves by tracking the two optimal values, which is the value founded by particles themselves and the value founded by the entire population so far. After finding the two values, particles update their velocity and position according to the following formula[6] :

$$
\begin{gathered}
v_{i d}(\text { new })=w \times v_{i d}(\text { old })+c_{1} \times r_{1} \times\left(p_{i d}-x_{i d}\right)+c_{2} \times r_{2} \times\left(p_{g d}-x_{i d}\right) \\
x_{i d}(\text { new })=x_{i d}(\text { old })+v_{i d}(\text { new })
\end{gathered}
$$




$$
w=w_{\max }-t \times\left(w_{\max }-w_{\min }\right) / \max D T
$$

In this formula, $w$ is constant of non-negative, we call it the inertia factor, which is used to balance the global searching and the local searching. Shi and other people for the first time introduce to update formula for the inertia weight of the speed in PSO[7]. In this paper, we propose $w_{\max }=0.9, w_{\min }=0.4[8] ; c_{1}=2.8, c_{2}=1.3 ; r_{1}$ and $r_{2}$ are random number in $[0,1], \max D T$ is the maximum number of iterations, in order to control the closing conditions of the algorithm; $t$ is the current number of iterations. In this paper, $w$ decrease gradually from $[0.9,0.4]$ [9].

Firstly, calculate the new speed of the particles by the distance of the original particle velocity and position between the two optimal locations according to the formula (1). Then particles move to a new location according to the formula (2). So back and forth. If it reached the specified number of iterations, the program terminates.

\subsection{The Processes of PSO}

(1) Initialize the initial position and velocity of a group of particles and set the number of iterations for 5000

(2) Set the mean square error as the fitness value and get the fitness value of each particle by calculating

(3) For every particle, compare it's fitness value with the best position (pbest,) if better than pbest, use it as the current optimal particle position (pbest);

(4) For every particle, compare it's pbest with the best position gbest of all particles, if better than gbest, use it as the current optimal particle position (gbest);

(5) According to the formula (1) and (2), adjust the speed and position of the particle;

(6) If it has not reach the end of the conditions, turn to step 2;

Here, the termination condition is that the required iterations can get the number of 5000 .

\section{Experimental Part}

\subsection{The Sources of Material}

In this paper, the tobacco data is $\mathrm{K} 326$ tobacco varieties, which is coming from the national 18 origins and is divided into three tobacco grades $(\mathrm{B} 2 \mathrm{~F}, \mathrm{X} 2 \mathrm{~F}, \mathrm{C} 3 \mathrm{~F})$.

\subsection{The Collection of Spectral Information}

In this paper, the spectra data is collected on five different parts of the tobacco leaf, and then take the average of the five parts as the spectrum. Tobacco original near infrared spectroscopy is showed in Figure 1. 


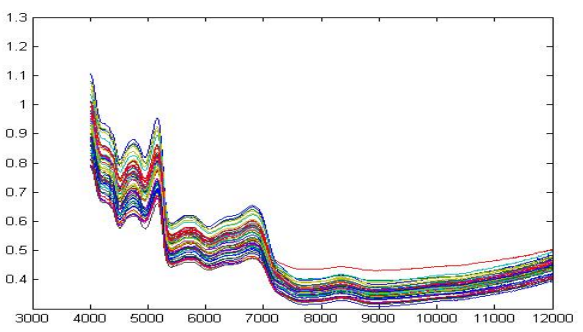

Fig. 1. Tobacco original near infrared spectroscopy

\subsection{The Preprocess of Spectral}

It can be seen from Figure 1, the main crest of tobacco information is concentrated in the 4000-9000 wavenumber. Therefore, in order to save modeling time, and eliminate the unwanted ingredients in the spectral information, in this paper, the wavenumber in the range of 4000-9000 $\mathrm{cm}^{-1}$ information are selected for the study.

The spectrum is showed in Figure 2 after wavelength selection. In this paper, use the first difference to eliminate the impact of the offset. The spectrum is showed in Figure 3 after the first difference. Then use the principal component analysis (PCA) to reduce the dimension and get the principal component of the spectrum. Use the PSO to create a system model. In the model, the main components of the spectral data is as the variable input of the model, the chemical values of nicotine and total nitrogen content is as the output of the model [10]. Use the data of mean square error between the calculated results and the real chemical value as the fitness function, in order to adjust the speed and position of the particle in the particle swarm and determine the global optimal solution. In the paper, the number of the principal components, the range of chemical value content of nicotine and the total nitrogen in modeling and in the prediction are showed in Table 1.

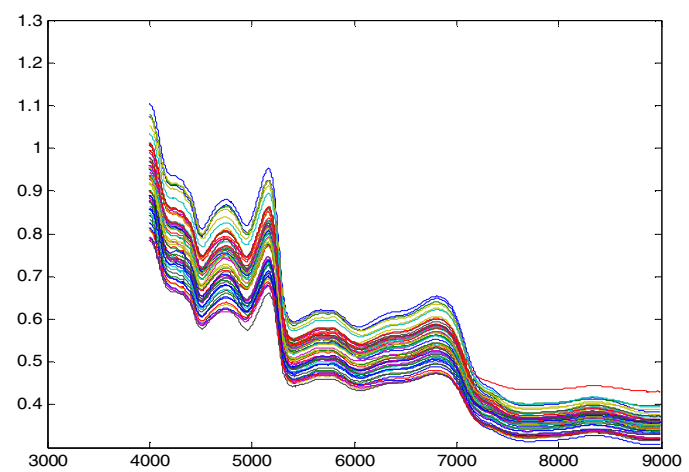

Fig. 2. Spectra after the wavelength selection 


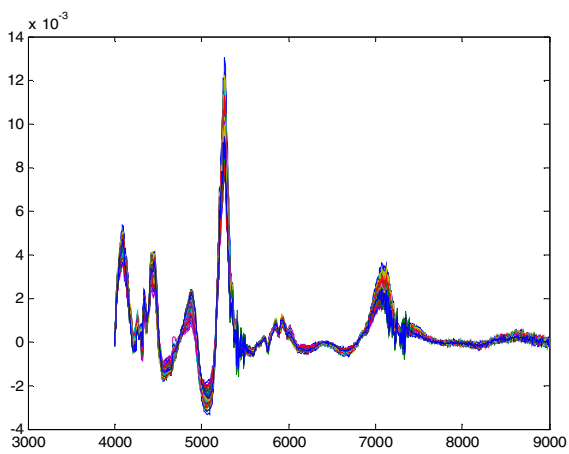

Fig. 3. Spectra after first-order Differential

Table 1. Range of chemical content and the number of principal components

\begin{tabular}{ccccc}
\hline $\begin{array}{l}\text { Sample } \\
\text { Name }\end{array}$ & $\begin{array}{c}\text { Samples } \\
\text { number }\end{array}$ & $\begin{array}{c}\text { The range of } \\
\text { model } \\
(\%)\end{array}$ & $\begin{array}{c}\text { The } \\
\text { range of } \\
\text { prediction } \\
(\%)\end{array}$ & $\begin{array}{r}\text { Principal } \\
\text { components }\end{array}$ \\
\hline $\begin{array}{c}\text { Nicotine } \\
\text { total }\end{array}$ & 57 & $0.88-4.68$ & $0.94-4.67$ & 14 \\
nitrogen & 57 & $1.45-3.83$ & $1.58-3.55$ & 15 \\
\hline
\end{tabular}

\subsection{The Parameter Settings of the Model}

In PSO, the parameter setting is very important. In order to improve the ability of modeling and prediction, in this paper, the algorithm parameter settings are showed in Table 2.

Table 2. The parameter settings of the model

\section{The parameter settings of the model}

The location of the particles (X)

Random

The velocity of the particle (V)

Random, but between[-1,1]

Maximum number of iterations (MAXDT)

5000

Number of particles $(\mathrm{N})$

50

$\mathrm{c} 1, \mathrm{c} 2$

$\mathrm{c} 1=2.8, \mathrm{c} 2=1.3$

\subsection{The Establishment and Evaluation of the Model}

In this paper, in all of $57 \mathrm{~K} 326$ tobacco data, use 40 samples as the set of modeling, 17 samples as the set of prediction. In the model, the total nitrogen modeling set of 
correlation coefficient is 0.9290 , the prediction set of correlation coefficient is 0.8487 ; nicotine modeling set of correlation coefficient is 0.8886 , the correlation coefficient of prediction set is 0.8786 . Other related indicators of the rest of the model are showed in Table 3. Figure 4 is PSO regression of nicotine modeling set and prediction set. Figure 5 is PSO regression of total nitrogen modeling set and prediction set.

Table 3. The indicators of evaluating the model

\begin{tabular}{ccccc}
\hline $\begin{array}{c}\text { Sample } \\
\text { Name }\end{array}$ & Modeling R & $\begin{array}{c}\text { Modeling } \\
\text { RMSEC }\end{array}$ & Prediction R & $\begin{array}{c}\text { Prediction } \\
\text { RMSEP }\end{array}$ \\
\hline $\begin{array}{c}\text { Nicotine } \\
\text { total } \\
\text { nitrogen }\end{array}$ & 0.8886 & 0.4737 & 0.8786 & 0.6417 \\
\hline
\end{tabular}
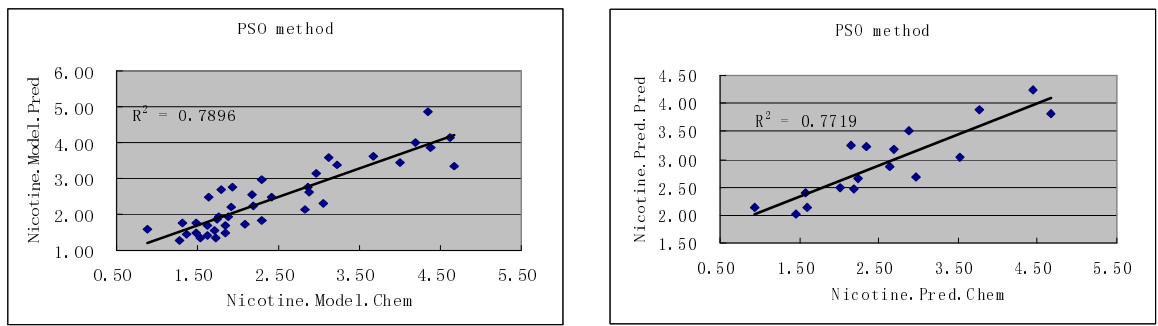

Fig. 4. PSO regression of nicotine modeling set and prediction set
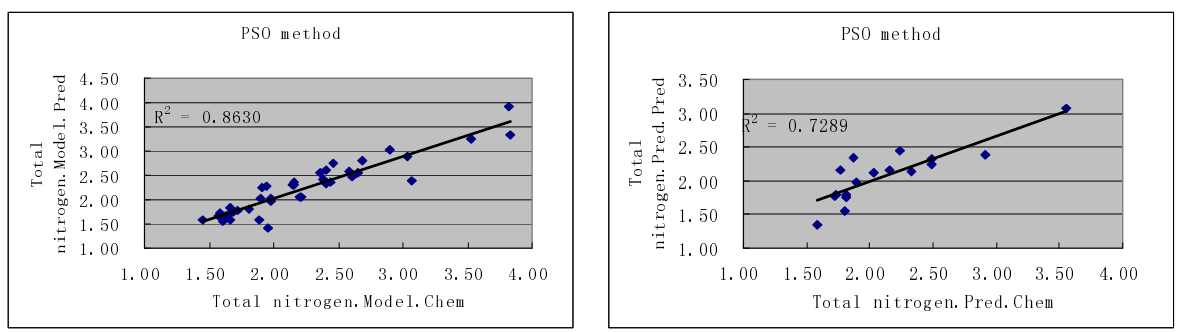

Fig. 5. PSO regression of total nitrogen modeling set and prediction set

\section{Results and Discussion}

57 tobacco samples were chosen, the near-infrared diffuse reflectance spectra at five different parts of tobacco leafs were averaged as the original spectra, the range of 4000-9000 wavenumber of spectral information was selected after wavelength selection, first-order differential was used to eliminate the impact of offset. PCA was used to reduce the dimensions and select the most representative of the principal components of the sample information. After these, use PSO and the data of chemical 
composition of nicotine and total nitrogen, to establish the models of near-infrared spectra of the main ingredient in tobacco quantitative analysis. In which, nicotine and total nitrogen quantitative analysis of modeling set correlation coefficients were $0.8886,0.9290$; the prediction set correlation coefficients were $0.8786,0.8487$. RMSEC values were $0.4737,0.2215$; RMSEP values were $0.6417,0.2677$. The results show that: PSO can be used to establish the model of nicotine and total nitrogen by near infrared spectroscopy quantitative analysis in tobacco.

\section{References}

1. Heng, L.Y., Long, L.Z., Dong, H.H., et al.: China Light Industry Press, 1-6 (2005)

2. Yan, Q.D., Jia, J.W., et al.: Laser and Infrared 37, 1058-1061 (2007)

3. Xu, L.H., Cai, C., et al.: Spectroscopy and Spectral Analysis 27, 2460-2463 (2007)

4. Yong, Z., Qian, C., et al.: Journal of Chemical 30, 697-700 (2009)

5. Kennedy, J., Eberhart, R.C.: Particle swarm optimization. In: Proceedings of the IEEE Conference on Neural Networks, pp. 1942-1948. IEEE Press, Washington, DC (1995)

6. Shao, R.H.: Computer Engineering and Design 30, 977-1980 (2009)

7. Shi, Y., Eberhart, R.C.: A modified particle swarm optimizer. In: Proceedings of IEEE International Conference on Evolutionary Computation, pp. 69-73. IEEE Press, Washington, DC (1998)

8. Jun, W.W., Ding, W.W.: Journal of Systems Engineering 20, 194-198 (2005)

9. Jiu, F.X., Guo, L.M., Jin, R.N., Xiao, T.C.: He'nan Institute of Technology (Natural Science) 22, 68-72 (2010)

10. Xin, J.W., Yuan, L., Dan, Z., Chong, E.T.: Liaoning University of Technology 34, 18-20 (2007) 